\title{
The Burden of Hypercoagulability in COVID-19
}

\author{
Madeleine Kim ${ }^{1}$ Andrew George ${ }^{2}$ Latha Ganti ${ }^{30}$ Derrick Huang ${ }^{4}$ Matthew Carman ${ }^{5}$
}

${ }^{1}$ The Baylor School, Chatanooga, Tennessee, United States

2 Department of Biology and Medicine, Brown University, Providence,

Address for correspondence Latha Ganti, MD, MS, MBA, University of Rhode Island, United States

${ }^{3}$ Department of Emergency Medicine, University of Central Florida, Central Florida, 6850 Lake Nona Boulevard Orlando, Orlando, Florida Orlando, Florida, United States

${ }^{4}$ Department of Emergency Medicine, University of Central Florida 32816-2368, United States (e-mail: lathagantimd@gmail.com). College of Medicine, Orlando, Florida, United States

${ }^{5}$ Department of Emergency Medicine, Lakeland Regional Health

Medical Center, Lakeland, Florida, United States

TH Open 2022;6:e96-e98.

\begin{abstract}
The novel coronavirus disease 2019 (COVID-19) infection has widespread impact on multiple organ systems, including damage to endothelial cells. Various studies have found evidence for direct mechanisms by which interaction between severe acute respiratory syndrome-coronavirus-2 (SARS-CoV-2) and endothelial cells lead to extensive damage to the latter, and indirect mechanisms, such as excessively elevated

Keywords

- pulmonary embolism

- COVID-19

- SARS-CoV-2

- deep venous thrombosis cytokines, can also result in the same outcome. Damage to the endothelium results in release of thrombotic factors and inhibition of fibrinolysis. This confers a significant hypercoagulability burden on patients infected or recovering from COVID-19 infection. In this case report, the authors report the case of a gentleman presenting with extensive deep vein thrombosis and pulmonary embolism, in the context of recent COVID-19 infection. The postulated mechanisms and management are discussed.
\end{abstract}

\section{Introduction}

The novel coronavirus disease 2019 (COVID-19) was designated as a global pandemic by the World Health Organization on March 11, 2020. COVID-19 is also strongly associated with thrombotic complications, such as deep vein thrombosis (DVT) and pulmonary embolism (PE) which are highly concerning due to associated poorer outcomes. ${ }^{1}$ In the emergency department (ED), patients presenting with extremity pain are assessed for risk factors for thrombotic complications, such as a history of DVT, PE, recent surgeries, and active cancer. However, in the setting of COVID-19, patients without traditional risk factors may still be at high risk for thrombosis. The authors present a case of thrombosis in a patient without traditional hypercoagulable risk factors and discuss a review of recent literature on the pathophysiology behind COVID-19 and hypercoagulability.

received

January 1, 2022

accepted

January 20, 2022

\section{Case Presentation}

A 62-year-old male presented to the emergency department due to right leg pain for 2 days. He elected not to receive the COVID-19 vaccine, and contracted COVID-19 pneumonia 10 days prior, and had been convalescing at home with antibiotics and prednisone. He denied any fevers, chills chest pain, shortness of breath, nausea, vomiting, diarrhea, abdominal pain, headache, or urinary symptoms. The patient had elevated the leg, taken some over-the-counter analgesics, and tried to rest it. However, it had progressively gotten more swollen and painful. The morning of his ED visit, he noticed that the outline of the veins were actually visible, something he had not previously seen (-Fig. 1). He also noted that the veins were very hard to touch, in contrast to veins that one can compress. He described the leg as being so painful now that it was hurting even to set it on the floor. The

\section{(C) 2022. The Author(s).}

This is an open access article published by Thieme under the terms of the Creative Commons Attribution License, permitting unrestricted use, distribution, and reproduction so long as the original work is properly cited. (https://creativecommons.org/licenses/by/4.0/)

Georg Thieme Verlag KG, Rüdigerstraße 14, 70469 Stuttgart, Germany 


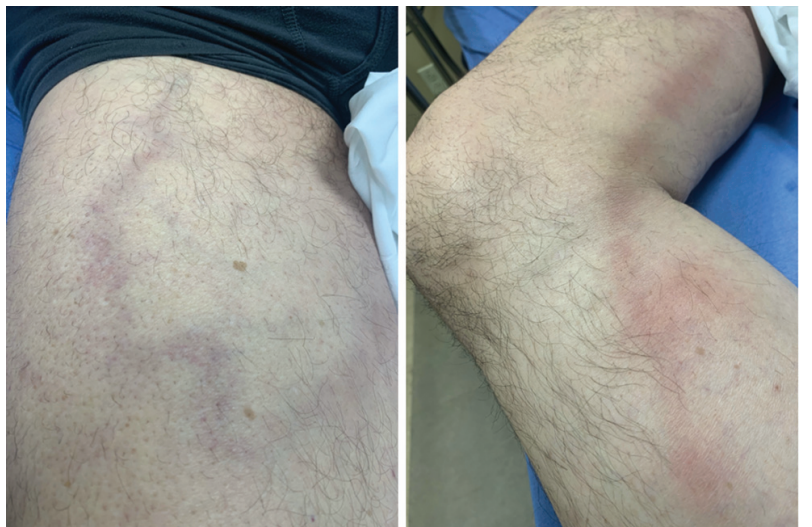

Fig. 1 Picture of the patient's leg showing the outline of the painful vein.

patient had no past medical history besides his COVID-19 illness, and was a nonsmoker. He was also physically quite fit, exercising daily, and working as a martial arts teacher.

On physical examination, his temperature was $98.2^{\circ} \mathrm{F}$, pulse 94 beats per minute, respirations 16 breaths per minute, blood pressure $118 / 80 \mathrm{~mm} \mathrm{Hg}$, and his oxygen saturation was $92 \%$ on room air. His heart, lungs, abdomen, and neurological status were all unremarkable. Examination of the right leg revealed it to be tender to palpation, mildly edematous, mildly erythematous, and with the outline of the veins clearly visible. The veins were also locally tender to palpation and somewhat hard.

Duplex venous ultrasonography revealed DVT in the right popliteal and calf veins and superficial vein thrombosis of the greater saphenous vein. Computed tomography angiography scan of the chest revealed left lower lobe pulmonary emboli and extensive bilateral patchy ground-glass airspace opacities ( - Fig. 2A and B). The patient was started on heparin and admitted to the hospitalist service. During hospitalization, his platelet counts rose as high as $562,000 / \mathrm{mm}^{3}$, prompting a hematology consult. They performed a myeloproliferative disease workup which was negative. The elevated platelet count was attributed to a reactive thrombosis. The patients was discharged with a direct oral anticoagulant.

\section{Discussion}

Though the mechanisms are not fully known, emerging evidence through recent months regarding the effects of severe acute respiratory syndrome-coronavirus-2 (SARS-CoV-2) infection suggests a state of hypercoagulability associated with COVID-19. ${ }^{1}$ Thrombotic complications overall have been a hallmark of COVID-19, with some studies reporting an incidence rate of such complications as high as $79 \%$, even among otherwise healthy, young individuals presenting with the disease. ${ }^{2}$ Even more conservative estimates find a significant incidence of adverse thrombotic events in COVID-19 patients, with the absolute best-case scenario rating, the risk at around 9\% in non-intensive care unit (ICU) patients (the same study reported a risk of $59 \%$ in ICU patients). ${ }^{3}$

Common laboratory hallmarks of COVID-19 have been known almost since the initial emergence of the disease to include elevated D-dimers, associated with a poor prognosis. ${ }^{4}$ Additional markers include elevated fibrinogen, low platelet counts, and prolonged coagulation times. ${ }^{5}$

Recent efforts have resulted in a few different mechanisms being proposed to explain the hypercoagulability state observed in COVID-19 patients. Such explanations seek to provide a link between observed features of COVID-19 and their interaction with the coagulation pathway. Cytokine alterations, endothelial cell injury, platelet activation, neutrophil extracellular traps (NETs), complement activation, and hypoxia all provide, potential explanations, either taken individually or as part of a complex system of interactions and modulation driving the hypercoagulability state.

The first of these comes from the observation of an elevation in proinflammatory cytokines within COVID-19 patients. Of note, key players in the extrinsic coagulation pathway (upstream activators of tissue factor [TF] release) interleukin (IL)-1, IL-6, and tumor necrosis factor (TNF)- $\alpha$ are
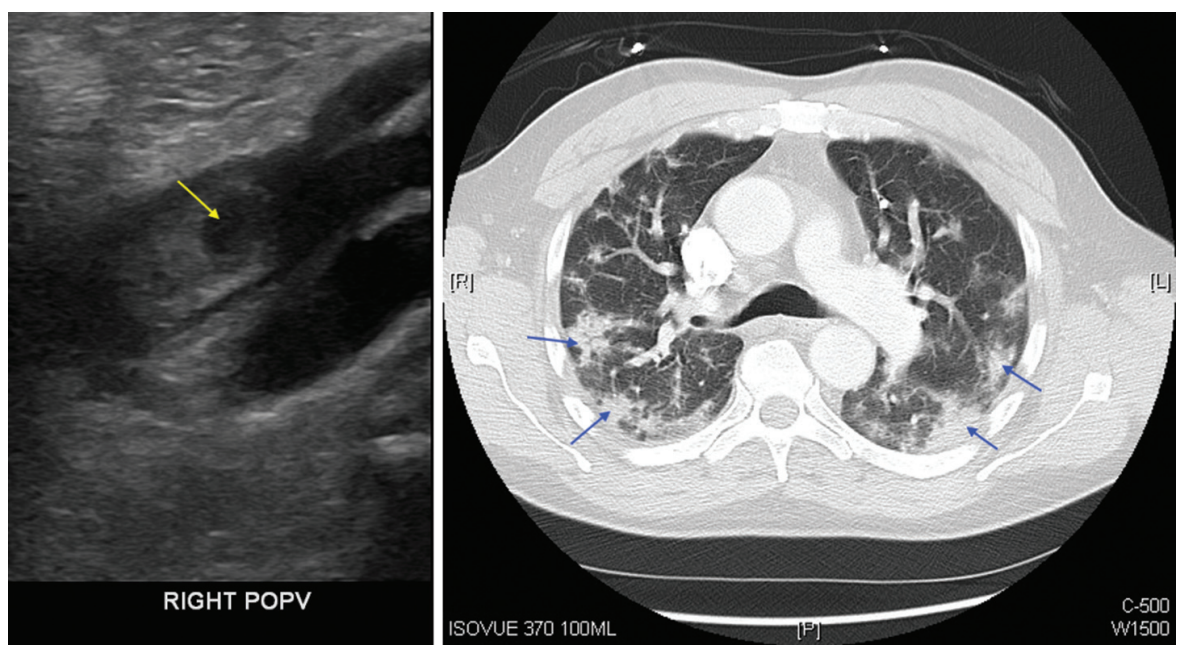

Fig. 2 Left panel shows DVT on ultrasonography (yellow arrow); right panel shows CT with ground glass opacities (blue arrows). CT, computed tomography; DVT, deep vein thrombosis. 
often elevated in COVID-19 patients. ${ }^{6}$ The same cytokines additionally suppress fibrinolysis secondary to increased Plasminogen Activator Inhibitor (PAI)-1 expression. ${ }^{7}$ Furthermore, the overall inflammatory state is well known to reduce TF pathway inhibitors, driving coagulation, and potentially working through factor (F)-Xa to enhance inflammation. ${ }^{8}$ Finally, such elevated cytokines likely contribute to other purported drivers of hypercoagulability such as NETs and phospholipid responses, discussed in further details shortly.

It is well-reported that COVID-19 can cause damage to endothelial cells. Various studies have found evidence for direct mechanisms by which interaction between SARS$\mathrm{CoV}-2$ and endothelial cells lead to extensive damage to the latter, and indirect mechanisms, such as excessively elevated cytokines, can also result in the same outcome. ${ }^{9,10}$ Damage to the endothelium results in release of thrombotic factors and inhibition of fibrinolysis.

As discussed previously, low platelet counts are a common sign of COVID-19. While the exact reason is unknown (Xu et al proposed three mechanisms: (1) virus-driven decrease in platelet synthesis, (2) virus-driven increased platelet destruction, and (3) virus-driven thrombosis to deplete platelets), ${ }^{11}$ the consequence is generally a high degree of platelet activation among remaining platelets due to microthrombi formation, contributing to hypercoagulability. ${ }^{12,13}$ Additionally, factors, such as hypoxia common in COVID-19, contribute to platelet activation, along with other hypoxia inducible factor (HIF)- $1 \alpha$ mediated prothrombotic and antifibrinolytic effects. ${ }^{14-16}$

The state of inflammation seen in COVID-19 is also conducive to NETs, used by neutrophils to target pathogens. NETs have been reported to be present in COVID-19 patients, and recent studies have found an association between hypercoagulability and NETs. ${ }^{17}$ Indeed, NETs tend to activate the vascular endothelium and platelets to drive thrombosis. ${ }^{18}$ Various studies have also linked COVID-19 to elevated complement activation and found associations between elevated complement activation and thrombosis in patients with severe COVID-19. ${ }^{19,20}$ Such a role is perhaps unsurprising, given the nature of the complement system and its tendency to drive microthrombi formation, as well as contribute to neutrophil activation and endothelial cell damage, which themselves have been proposed to drive hypercoagulability in COVID-19 patients.

\section{Conclusion}

Ultimately, whether it is one of these mechanisms or something yet to be determined, the clinical outcome of hypercoagulability is nevertheless a serious consideration when treating COVID-19 patients. As observed in this case report, such outcome does not discriminate for health prior to contracting COVID-19.

Conflict of Interest

None declared.

\section{References}

1 Kichloo A, Dettloff K, Aljadah M, et al. COVID-19 and hypercoagulability: a review. Clin Appl Thromb Hemost 2020; 26:1076029620962853

2 Nahum J, Morichau-Beauchant T, Daviaud F, et al. Venous thrombosis among critically ill patients with coronavirus disease 2019 (COVID-19). JAMA Netw Open 2020;3(05):e2010478

3 Middeldorp S, Coppens M, van Haaps TF, et al. Incidence of venous thromboembolism in hospitalized patients with COVID-19. J Thromb Haemost 2020;18(08):1995-2002

4 Tang N, Li D, Wang X, Sun Z. Abnormal coagulation parameters are associated with poor prognosis in patients with novel coronavirus pneumonia. J Thromb Haemost 2020;18(04):844-847

5 Han H, Yang L, Liu R, et al. Prominent changes in blood coagulation of patients with SARS-CoV-2 infection. Clin Chem Lab Med 2020; 58(07):1116-1120

6 Mehta P, McAuley DF, Brown M, Sanchez E, Tattersall RS, Manson JJHLH Across Speciality Collaboration, UK. COVID-19: consider cytokine storm syndromes and immunosuppression. Lancet 2020;395(10229):1033-1034

7 Sawdey MS, Loskutoff DJ. Regulation of murine type 1 plasminogen activator inhibitor gene expression in vivo. Tissue specificity and induction by lipopolysaccharide, tumor necrosis factor-alpha, and transforming growth factor-beta. J Clin Invest 1991;88(04): 1346-1353

8 Jose RJ, Manuel A. COVID-19 cytokine storm: the interplay between inflammation and coagulation. Lancet Respir Med 2020;8(06):e46-e47

9 Varga Z, Flammer AJ, Steiger P, et al. Endothelial cell infection and endotheliitis in COVID-19. Lancet 2020;395(10234):1417-1418

10 Leisman DE, Deutschman CS, Legrand M. Facing COVID-19 in the ICU: vascular dysfunction, thrombosis, and dysregulated inflammation. Intensive Care Med 2020;46(06):1105-1108

11 Xu P, Zhou Q, Xu J. Mechanism of thrombocytopenia in COVID-19 patients. Ann Hematol 2020;99(06):1205-1208

12 Chen N, Zhou M, Dong X, et al. Epidemiological and clinical characteristics of 99 cases of 2019 novel coronavirus pneumonia in Wuhan, China: a descriptive study. Lancet 2020;395 (10223):507-513

13 Hottz ED, Bozza FA, Bozza PT. Platelets in immune response to virus and immunopathology of viral infections. Front Med (Lausanne) 2018;5:121

14 Cameron SJ, Mix DS, Ture SK, et al. Hypoxia and ischemia promote a maladaptive platelet phenotype. Arterioscler Thromb Vasc Biol 2018;38(07):1594-1606

15 Frangou E, Chrysanthopoulou A, Mitsios A, et al. REDD1/autophagy pathway promotes thromboinflammation and fibrosis in human systemic lupus erythematosus (SLE) through NETs decorated with tissue factor (TF) and interleukin-17A (IL-17A). Ann Rheum Dis 2019;78(02):238-248

16 Pugh CW, Ratcliffe PJ. New horizons in hypoxia signaling pathways. Exp Cell Res 2017;356(02):116-121

17 Zuo Y, Yalavarthi S, Shi H, et al. Neutrophil extracellular traps in COVID-19. JCI Insight 2020;5(11):138999

18 Zhang S, Zhang J, Wang C, et al. COVID-19 and ischemic stroke: mechanisms of hypercoagulability (review). Int J Mol Med 2021; 47(03):21

19 Riedl M, Fakhouri F, Le Quintrec M, et al. Spectrum of complement-mediated thrombotic microangiopathies: pathogenetic insights identifying novel treatment approaches. Semin Thromb Hemost 2014;40(04):444-464

20 Cugno M, Meroni PL, Gualtierotti R, et al. Complement activation in patients with COVID-19: a novel therapeutic target. J Allergy Clin Immunol 2020;146(01):215-217 